\title{
Spatio-temporal detection of Kelvin waves in quantum turbulence simulations
}

\author{
P. Clark di Leoni ${ }^{1}$, P.D. Mininni ${ }^{1}$, \& M.E. Brachet ${ }^{2}$ \\ ${ }^{1}$ Departamento de Física, Facultad de Ciencias Exactas y Naturales, \\ Universidad de Buenos Aires and IFIBA, CONICET, \\ Ciudad Universitaria, 1428 Buenos Aires, Argentina. \\ ${ }^{2}$ Laboratoire de Physique Statistique de l'Ecole Normale Supérieure associé au CNRS et aux Universités Paris 6 et 7 , \\ 24 Rue Lhomond, 75237 Paris Cedex 05, France.
}

(Dated: July 27, 2018)

\begin{abstract}
We present evidence of Kelvin excitations in space-time resolved spectra of numerical simulations of quantum turbulence. Kelvin waves are transverse and circularly polarized waves that propagate along quantized vortices, for which the restitutive force is the tension of the vortex line, and which play an important role in theories of superfluid turbulence. We use the Gross-Pitaevskii equation to model quantum flows, letting an initial array of well-organized vortices develop into a turbulent bundle of intertwined vortex filaments. By achieving high spatial and temporal resolution we are able to calculate space-time resolved mass density and kinetic energy spectra. Evidence of Kelvin and sound waves is clear in both spectra. Identification of the waves allows us to extract the spatial spectrum of Kelvin waves, clarifying their role in the transfer of energy.
\end{abstract}

\section{INTRODUCTION}

Quantum turbulence is the chaotic and erratic spatiotemporal behavior observed in superfluids and BoseEinstein condensates (BECs) [1, 2]. Its motion is characterized by the interaction between vortex filaments, where all vorticity is concentrated and which have quantized circulation [3]. As superfluids have no viscosity, quantum turbulence has garnered much attention from the classical turbulence community, for it could provide insight into extremely developed turbulence. But it has also been a matter of debate how similar quantum and classical turbulence actually are. One of the characteristical features of turbulence, the existence of a Kolmogorov energy spectrum, has been confirmed in superfluids [4, 5]. However, the physical mechanisms behind this spectrum are not completely understood. Moreover, other features, such as velocity statistics, appear to be different between classical and quantum turbulence [6, 7].

An interesting property of classical and quantum vortex lines, first predicted by Lord Kelvin [8], is that waves can propagate along them when the line is subjected to helical deformations. These waves are known as Kelvin waves. In quantum fluids they are believed to play a crucial role in the turbulent energy cascade 99 -12, where energy is transfered from large to small scales and it is ultimately dissipated by phonon emission [13, 14]: while at large scales vortex interaction and reconnection [15] dominate the transfer of energy, at small scales Kelvin waves are believed to interact nonlinearly exciting fluctuations at even smaller scales. They play a role in various problems in classical fluid dynamics [16, 17], but not in classical turbulence, being the turnover time or the period of waves particular to that system (such as inertial waves in a rotating flow, or Alfvén waves in a magnetofluid) the only relevant timescales [18, 19]. As a result, detection of Kelvin waves in a disorganized superfluid flow is considered a signature of quantum turbulence [6, 20].

There are several theoretical studies of Kelvin wave turbulence in superfluids, whose main focus is to understand nonlinear interactions and the resulting energy spectrum. Particular attention is given to the latter, as predictions for the energy spectrum differ. Using wave turbulence theory [21] different teams of researchers arrived to different answers, one being that the energy spectrum scales with wavenumber as $\sim k^{-7 / 5}$ [9], and the other that it goes as $\sim k^{-5 / 3}[10$ ] (it should also be noted that some authors claim that Kelvin waves play a neglible role in the spatial energy spectrum [22]). Recent numerical studies yield results compatible with a $\sim k^{-5 / 3}$ spectrum [23 26]. However, studies of wave dynamics usually have a single or a few vortices, either using vortex line dynamics simulations [25], or solving the Gross-Pitaevskii equation (GPE) [27]. In the laboratory Kelvin waves have been detected in superfluid helium using submicron ice particles as tracers [20]; the analysis focused on selected reconnection events and the subsequent emission of Kelvin waves. Finally, Kelvin waves and other vortex wave modes have also been studied and identified in BECs 28 31.

Our aim is to study Kelvin waves in quantum turbulence. As mentioned above, numerical studies of quantum turbulence have two main approaches. One is to simulate quantum vortex lines dynamics [26, 32]; in this approach the velocity field outside the vortex lines is given by the Biot-Savart law and reconnection events are performed ad-hoc. The approach we use solves the GPE, an equation for the evolution of the wavefunction $\psi$ for a system of bosons. A Kolmogorov spectrum $\sim k^{-5 / 3}$ was obtained in simulations of the GPE in [13, 33 35]. We consider a flow that shares similarities with the von Kármán flow generated in recent experiments with superfluid helium 36]. We extract and save four-dimensional information to compute the spatio-temporal spectrum of quantum turbulence, where the presence of sound and Kelvin waves is evident. Previous studies using a similar technique observed sound waves [37, 38], and Kelvin waves in a single and straight vortex filament [25]. Our 
study is done in a three-dimensional highly turbulent environment, with a large number of vortices.

\section{THE GROSS-PITAEVSKII EQUATION}

The GPE describes the evolution of the wavefunction $\psi$ of a field of weakly interacting bosons of mass $m$,

$$
i \hbar \frac{\partial \psi}{\partial t}=-\frac{\hbar^{2}}{2 m} \nabla^{2} \psi+g|\psi|^{2} \psi,
$$

where $g$ is proportional to the scattering length. By means of the Madelung transformation

$$
\psi(\mathbf{r}, t)=\sqrt{\frac{\rho(\mathbf{r}, t)}{m}} e^{i m \phi(\mathbf{r}, t) / \hbar},
$$

where $\rho$ is the density of particles and the phase $\phi$ can be associated with a velocity by $\mathbf{v}=\boldsymbol{\nabla} \phi$, one obtains a hydrodynamic description of the system [39],

$$
\begin{gathered}
\frac{\partial \rho}{\partial t}+\nabla \cdot(\rho \mathbf{v})=0 \\
\frac{\partial \mathbf{v}}{\partial t}+\mathbf{v} \cdot \nabla \mathbf{v}=-\frac{g}{m^{2}} \nabla \rho+\frac{\hbar^{2}}{2 m^{2}} \nabla\left(\frac{\nabla^{2} \sqrt{\rho}}{\sqrt{\rho}}\right) .
\end{gathered}
$$

These equations are similar to the Euler equations for a classical and compressible barotropic fluid, except for the extra second term on the r.h.s. of Eq. (4) which is referred to as the "quantum pressure". By solving the GPE we get the full three dimensional velocity and density fields, and compared to other methods [40], we do not need to reconstruct them from the vortex tangle configuration.

The hydrodynamic description allows us to define a quantity akin to the classical kinetic energy of a fluid, namely $E_{k}=\rho v^{2} / 2$. Note that this is only a fraction of the total energy density, given by $\hbar^{2}|\nabla \psi|^{2} / 2 m+g|\psi|^{4} / 2$. For simplicity, we will refer to the classical kinetic energy just as the kinetic energy. It can be further decomposed into an incompressible component $E_{k}^{i}$ and a compressible component $E_{k}^{c}$, by decomposing the velocity field into irrotational and solenoidal components. The two components will be useful to discriminate between sound waves and other excitations in the fluid. More details on the energy decompositions are given below (see also [33, 41]).

This system can have sound waves which follow the Bogoliubov dispersion relation [39],

$$
\omega_{B}(k)=k \sqrt{c^{2}+\frac{c^{2} \xi^{2}}{2} k^{2}},
$$

where the speed of sound is $c=\sqrt{g|\psi|^{2} / m}$ and the coherence length is $\xi=\sqrt{\hbar^{2} /\left(2 m|\psi|^{2} g\right)}[33$, [39], and Kelvin waves [3] which follow the dispersion relation

$$
\omega_{K}(k)=\frac{2 c \xi}{\sqrt{2} a^{2}}\left(1 \pm \sqrt{1+k a \frac{K_{0}(k a)}{K_{1}(k a)}}\right),
$$

where $a$ is the vortex core radius, and $K_{0}$ and $K_{1}$ are modified Bessel functions. The dispersion relation is quadratic in the small $k$ limit, while for large $k$ it is linear.

To solve numerically Eq. (1) we use GHOST [42], a highly parallel code which uses a pseudospectral method to compute spatial derivatives, fourth-order RungeKutta to compute time derivatives, and can solve PDEs in Cartesian periodic grids. We use $512^{3}$ grid points and the " $2 / 3$ rule" for de-aliasing. The speed of sound is $c=2$ and the coherence length is $\xi=0.1 /(8 \sqrt{2})$ in dimensionless units in a three-dimensional box of length $L=2 \pi$. Quantities are made dimensionless using characteristic length $L_{0}$, velocity $U_{0}$, and mean density $\rho_{0}$ (see [33]). These parameters result in an intervortex distance $\ell$ such that $k_{\ell}=2 \pi / \ell \approx 10$ (see [13, 33]), and in a vortex core radius $a \approx 2 \xi$ as measured directly from the full width at half maximum of the mass density profile. Also, the quantum of circulation $h / m$ in dimensionless units is given by $4 \pi c \xi / \sqrt{2}=0.05 \pi$.

As initial condition we use the Taylor-Green flow, which results in a set of vortex loops in two counterrotating large scale eddies with turnover time of order unity, and whose geometry mimics the von Kármán flow 33]. The von Kármán flow is used in recent experiments with two counter-rotating propellers such as SHREK (the Superfluid High REynolds von Kármán experiment) [36], and has been used in the past to measure Kolmogorov spectra in superfluids [4]. For more information about the generation of the initial conditions see Appendix $\mathrm{A}$. We let the simulation run for $\approx 20$ large-scale turnover times, so as to get good statistics on the slowest waves in the system. To also resolve the fastest waves, we use a very high output cadence, storing one output of the wavefunction every half period of the fastest waves (i.e., the sound waves for the maximum wavenumber in the spatial domain). This very high temporal resolution allows us to properly calculate space and time resolved spectra.

\section{CHARACTERIZATION OF THE FLOW}

Figure 1 shows a three-dimensional rendering of the density field $\rho$. The visualization is done using the software VAPOR [43], and only regions with low density (indicating topological defects associated with quantized vortices) are shown. The field evolves from a well ordered structure to a bundle of intertwined and structurally rich vortices. This evolution is associated with the development of reconnection events, which become prominent at $t \approx 5$ and start to subside after $t \approx 10$. The analysis below is done during this period, as afterwards too much of the total energy has decayed into phonons. Tracking reconnection events and the subsequent generation of Kelvin waves in this flow is not an easy task. However, the identification of helical waves from spatial observations is possible if single vortices or single reconnection events are followed in time (see, e.g., 20]).

In Fig. 5 we present the evolution of the different com- 
ponents of the total energy in the simulation. As mentioned above, the classical kinetic energy density is defined as

$$
E_{k}=\frac{1}{2} \rho v^{2}
$$

The remaining components of the total energy density in the system are the so-called quantum energy

$$
E_{q}=\frac{\hbar^{2}}{2 m^{2}}(\nabla \sqrt{\rho})^{2},
$$

and the potential (or internal) energy

$$
E_{p}=\frac{g}{2 m^{2}} \rho^{2}
$$

A detailed analysis and derivation of each component of the total energy density can be found, e.g., in [41].

From Fig. 5 different regimes can be identified in the evolution. In the first stage, up to $t \approx 5$, the incompressible kinetic energy oscillates around a mean value. Afterwards it decays and the other components of the energy grow, as reconnection of vortex lines takes place and the flow becomes more complex (see Fig. 1). After $t \approx 10$ the number of reconnection events in the flow subsides and the growth of the compressible, potential, and quantum energy becomes slower. During this process the total energy is conserved up to the sixth significant digit. Mass and momentum are also conserved. Snapshots of the velocity field and of the density between $t \approx 5$ and $\approx 10$, when the turbulent spectrum is more developed, are thus used for the spatio-temporal analysis.

\section{LOOKING FOR NEEDLES IN A HAYSTACK}

Instantaneous flow visualization is insufficient to identify and extract all the waves in a turbulent flow. In particular, in order to quantify their relevance in the energy cascade, it is necessary to quantify their amplitudes as a function of frequency and wavenumber, i.e., to calculate space-time resolved spectra. As Kelvin waves are oscillations of the lines with $\rho=0$, we should be able to identify their imprint in the mass spectrum, and we therefore study it first. Figure 2 shows the mass spectrum $\rho(k, \omega)$, along with a close-up for low wavenumbers, as well as a cut of $\rho(k, \omega)$ at $k=32$. The dashed line in Fig. 2 corresponds to the Bogoliubov linear dispersion relation of sound waves [Eq. (5)], while the solid line corresponds to that of Kelvin waves [Eq. (6) $]$. Substantial power is concentrated along these curves. At low wavenumbers Kelvin waves are dominant, whereas sound waves become prominent as Kelvin waves begin to fade for $k \gtrsim 50$. At very high wavenumbers another accumulation of energy can be seen for $\omega \approx 350$, which is probably due to quantum pressure effects inside the vortex core. Indeed, the spectrum of the quantum pressure also shows a bump at these wavenumbers (not shown), and

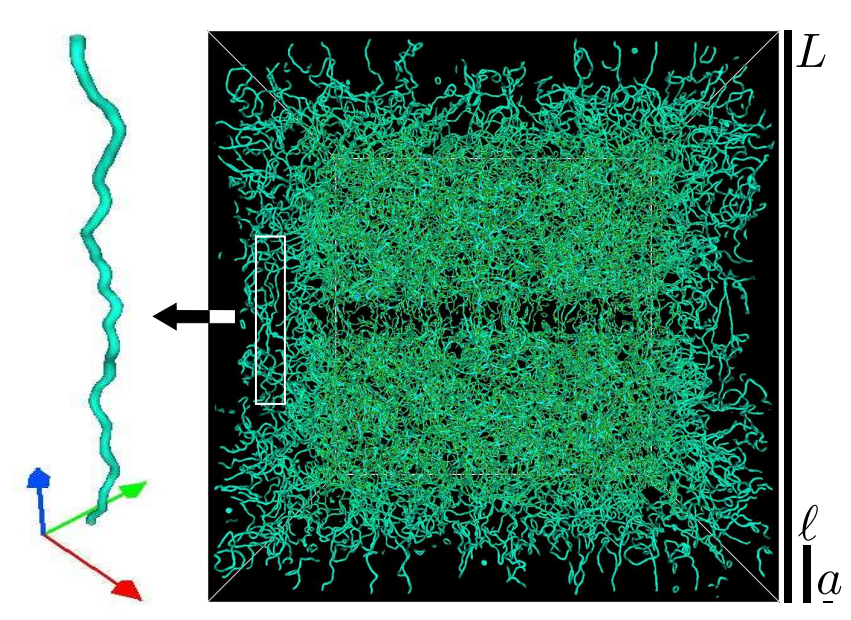

FIG. 1. (Color online) Three-dimensional rendering of the mass density. The lines, corresponding to regions of low density, are associated with quantized vortices. Typical length scales are indicated by the black bars (see text for description). On the left we show a zoom into a single vortex; helical perturbations propagating along it can be identified. Due to the highly turbulent nature of the flow, identifying waves here is like looking for needles in a haystack.

the frequencies are compatible with those predicted for axisymmetric oscillations of the vortex core [44, 45]. The presence of Kelvin waves at low wavenumbers is the most striking feature of the flow; these modes are probably excited by deformation of vortex lines by the large-scale flow, and are hard to identify by simple inspection of the evolution of individual vortex lines. In Appendix B, we present a benchmark sutdy of the spatio-temporal spectrum of mass in a flow where only linear Kelvin waves were excited, to verify the modes indentified in Fig. 2 correspond to these waves.

To independently verify the presence of sound and Kelvin waves, and to separate the multiple branches of the dispersion relation, we now consider the spectrum of the compressible kinetic energy $E_{k}^{c}(k, \omega)$, and of the incompressible kinetic energy $E_{k}^{i}(k, \omega)$ (see Fig. 3). A strong accumulation of energy around modes satisfying the relation $\omega=\omega_{B}(k)$ is evident in the compressible spectrum (Fig. 3A). In the incompressible spectrum (Fig. 3B) these excitations are negligible, but two new features are found. First, strong excitations can be observed for all wavenumbers at low frequencies. These excitations are compatible with sweeping of the vortex cores by the large-scale flow, i.e., the advection of small scale structures by the flow with a slow timescale associated with the turnover time. This is an important effect in classical turbulence where it is responsible for the temporal decorrelation of modes [18, 19]. It results in the smearing of the energy for all frequencies $\omega=U_{r m s} k$ and smaller (with r.m.s. velocity $U_{r m s} \approx 0.5$ ). Superposed to these excitations, modes compatible with Kelvin 


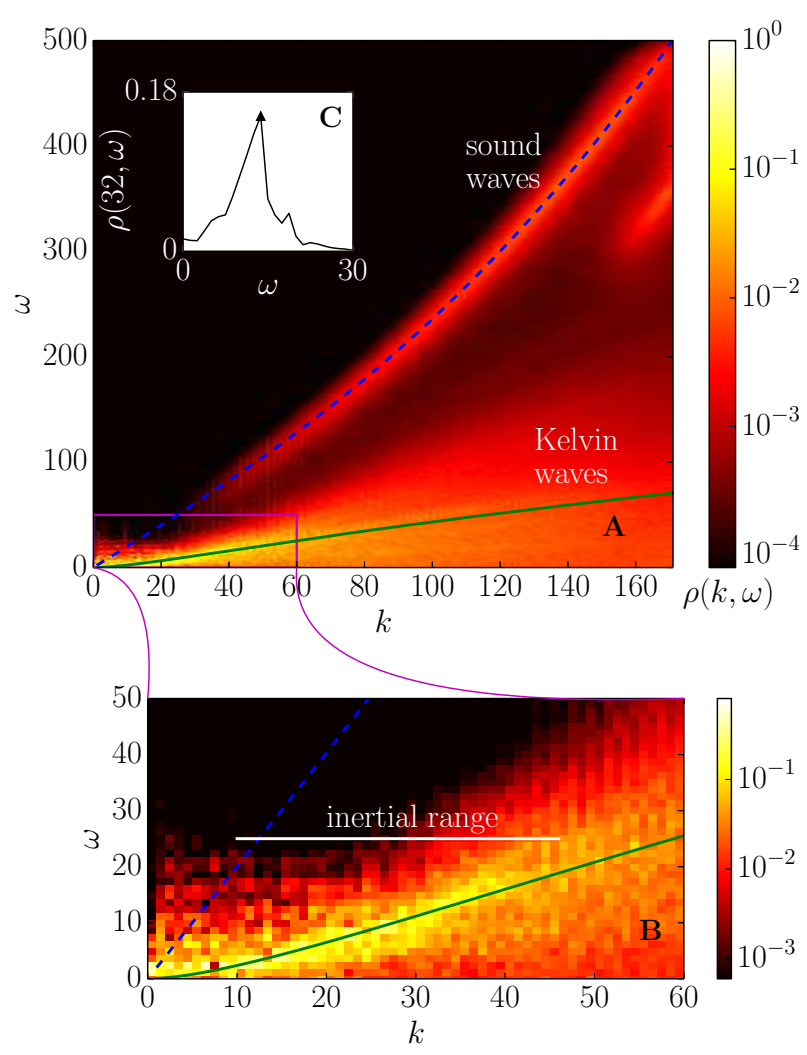

FIG. 2. (Color online) Space-time resolved mass spectrum, $\rho(k, \omega)$ normalized by $\rho(k)$. A: The whole spectrum; the dashed (blue) line indicates the sound wave dispersion relation $\omega_{B}(k)$, the solid (green) line indicates the Kelvin wave dispersion relation $\omega_{K}(k)$. B: Close-up for small wavenumbers; note the accumulation of power near the Kelvin wave modes. The range of wavenumbers with strong Kelvin excitations are marked as "inertial range". C: A cut of $\rho(k, \omega)$ (also normalized) for $k=32$, the marker indicates the Kelvin wave frequency. At low wavenumbers Kelvin waves are dominant, whereas sound waves become prominent after $k \approx 50$. At very high wavenumbers the quantum pressure inside the vortex core also leaves a trace in the spectrum.

waves can still be observed for small wavenumbers. To further verify this, we calculated the decorrelation time $\tau_{d}$ of individual Fourier modes and compared it to the sweeping time $\tau_{s} \sim 1 /\left(U_{r m s} k\right)$ and to the wave period $\tau_{\omega} \sim 1 / \omega_{K}(k)$ (Fig. 3). The decorrelation time fluctuates between $\tau_{s}$ and $\tau_{\omega}$ for low wavenumbers and converges towards $\tau_{s}$ for large wavenumbers; no other relevant time scales can be observed. Other collective vortex motions (e.g., Tkachenko waves [46 48]) may also be present but masked by these two dominant timescales. A weak trace of these effects is also present in $E_{k}^{c}(k, \omega)$. Kelvin waves do not contribute significant energy to the spectrum of classical turbulence, making the presence of energy in these modes a signature of quantum turbulence.

The role of Kelvin waves in the dynamics of quantum
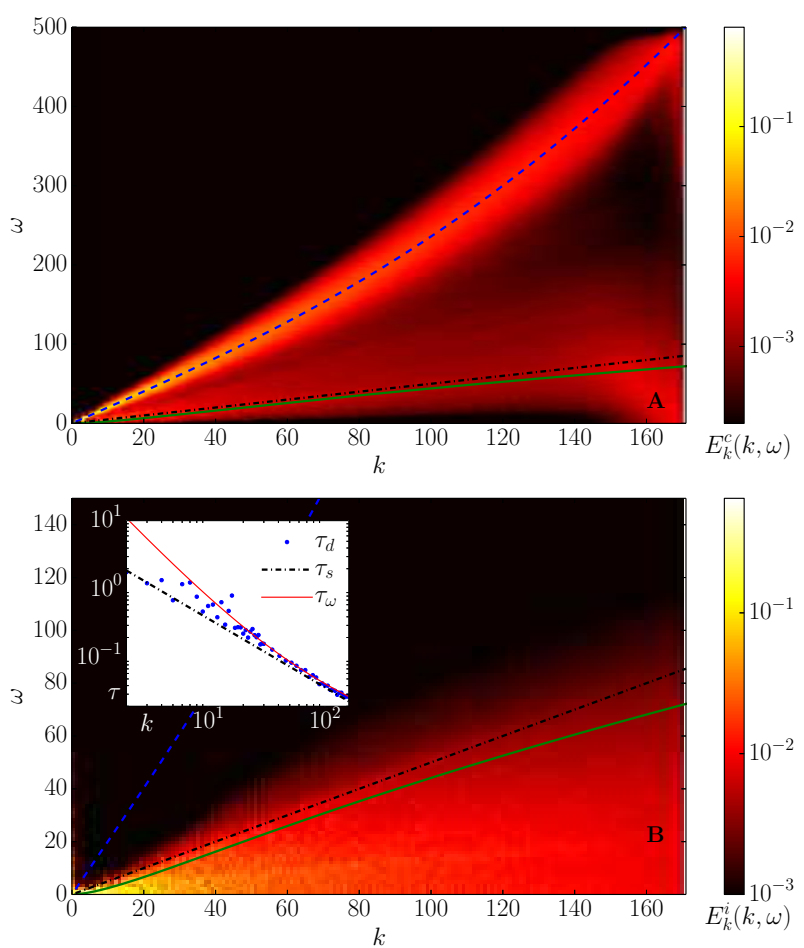

FIG. 3. (Color online) A: Space-time resolved compressible kinetic energy spectrum, $E_{k}^{c}(k, \omega)$, normalized by $E_{k}^{c}(k)$. B: Same for the incompressible kinetic energy spectrum $E_{k}^{i}(k, \omega)$. The dashed (blue) line corresponds to $\omega_{B}(k)$, and the solid (green) line corresponds to $\omega_{K}(k)$. The dash-dotted (black) line corresponds to sweeping, which excites all modes with frequency equal or smaller than $\omega=U_{r m s} k . E_{k}^{c}(k, \omega)$ is dominated by excitations around the sound wave dispersion relation while $E_{k}^{i}(k, \omega)$ shows sweeping and Kelvin wave excitations. Inset: decorrelation time $\tau_{d}(k)$ of individual Fourier modes, compared with the sweeping time $\tau_{s}$ (dash-dotted black line) and the Kelvin wave period $\tau_{\omega}$ (solid red line).

turbulence is controversial. While at large scales the interaction of quantized vortices with the flow results in advection and reconnection of vortex lines, at scales comparable to the intervortex distance $\ell$ Kelvin waves are believed to interact nonlinearly, exciting smaller fluctuations that eventually loose their energy to phonons. This transfer of energy by nonlinear interaction of wave modes can be described by wave turbulence theories, but current predictions differ on the shape of the energy spectrum [9, 10]. The quantification of the amplitude of all Kelvin waves modes shown in Figs. 2 and Fig. 3 can be used to shed some light on this problem. We thus extract the modes centered around the dispersion relation given by Eq. (6) with a width of $2 \sigma$ from the wave frequency, where the dispersion $\sigma$ is estimated by fitting the peaks in $\rho\left(k_{i}, \omega\right)$ such as the one shown in Fig. 2 $\mathrm{C}$ with a Gaussian. This subset of modes, which we call $\Omega_{K}$, can be used to compute the spectrum of the incompressible kinetic energy associated with Kelvin wave modes, $\int_{\Omega_{K}} E_{k}^{i}(k, \omega) \mathrm{d} \omega$ (Fig. 4). We verified that the shape of 


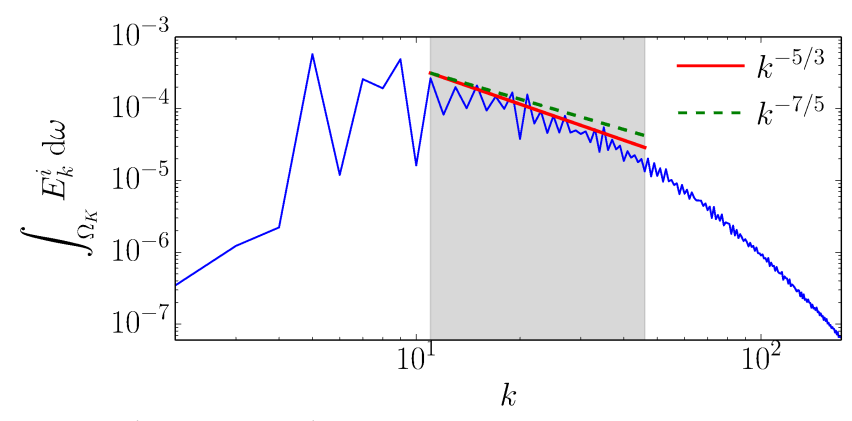

FIG. 4. (Color online) Spectrum of the incompressible kinetic energy associated with Kelvin wave modes, $\int_{\Omega_{K}} E_{k}^{i}(k, \omega) \mathrm{d} \omega$, where $\Omega_{K}$ are the modes neighboring $\omega_{K}(k)$. The two predictions for the Kelvin wave spectrum are shown as references. The shaded area corresponds to the region with strong Kelvin excitations identified as "inertial range" in Fig. 2]B

the spectrum is not very sensitive to the width in units of $\sigma$ used to define the subset $\Omega_{K}$, as long as it is not too large so as to include, e.g., modes associated with sound waves. In this spectrum, the candidate for an inertial range is observed for $k \gtrsim k_{\ell}$, and its width is in agreement with the wavenumbers for which we observe Kelvin-like excitations in Fig. 2, As the scale separation is limited, we do not attempt to fit the data in Fig. 4 and only show the two theoretical predictions for the scaling as references.

\section{CONCLUSIONS}

Kelvin wave turbulence is an inherently quantum regime of superfluids. Proper quantification of Kelvin waves is important to understand the differences between classical and quantum turbulence. We presented direct evidence of the presence of Kelvin waves in numerical simulations of quantum turbulence using the GPE. By looking at the space-time resolved mass density spectrum, we showed that Kelvin waves play the dominant role at scales comparable to the intervortex distance, while sound waves are excited at smaller scales. Furthermore, the kinetic energy spectrum confirms the presence of both waves in the flow. The presence of these waves in the spectrum can be considered a quantum signature that distinguishes this flow from classical turbulence.

Modes satisfying the wave dispersion relations excite a continuous region of the spectra, with broadening around the theoretical dispersion curves. This suggest that waves interact nonlinearly, as broadening of the dispersion relation in turbulent flows is often the result of nonlinear coupling and energy transfer. However, the modes excited are still close to the linear dispersion relations, an unexpected result as excitations in the turbulent regime do not necessarily have small amplitudes. Finally, extraction of the spectrum of the incompressible kinetic energy of modes along the dispersion relation of Kelvin waves results in a spectrum that is compatible with predictions of weak turbulence theories for superfluids.

\section{Appendix A: Preparation of the initial conditions}

The Taylor-Green initial conditions are generated by preparing a wavefunction $\psi$ whose associated velocity field is a Taylor-Green flow [33], given by

$$
\begin{aligned}
& v_{x}(x, y, z)=\sin (x) \cos (y) \cos (z), \\
& v_{y}(x, y, z)=-\cos (x) \sin (y) \cos (z), \\
& v_{z}(x, y, z)=0
\end{aligned}
$$

The Taylor-Green flow contained in a periodic box has properties that mimic the von Kármán flow driven by two counter-rotating impellers, and has been extensively used in simulations to compare with the experimental flow. The von Kármán flow is commonly used in the laboratory to study turbulence, including dynamo experiments with conducting flows, experiments to study Lagrangian particles, and superfluid turbulence 36.

The process to generate the initial wavefunction is described in great detail in 33]. Here we give a brief, but nonetheless complete, presentation. The Taylor-Green flow can be described by the Clebsch potentials

$$
\begin{aligned}
& \lambda(x, y, z)=\cos (x) \sqrt{2|\cos (z)|}, \\
& \mu(x, y, z)=\cos (y) \sqrt{2|\cos (z)|} \operatorname{sgn}[\cos (z)],
\end{aligned}
$$

which verify $\nabla \times \mathbf{v}=\nabla \lambda \times \nabla \mu$. Our use for them is that they can map a point in the $(\lambda, \mu)$ plane to a line in three-dimensional real space. Now, instead of having to assemble directly a three-dimensional wavefunction whose nodal lines match vortex lines of the velocity field $\mathbf{v}$, we can pick instead a complex field $\phi(\lambda, \mu)$ which has a zero (a defect) at the point $\left(\lambda_{d}, \mu_{d}\right)$. Then, the three-dimensional wavefunction

$$
\varphi(x, y, z)=\phi(\lambda(x, y, z), \mu(x, y, z)),
$$

will be equal to zero along the line(s) defined by $\lambda(x, y, z)=\lambda_{d}$ and $\mu(x, y, z)=\mu_{d}$. This ensures that the defects of $\varphi$ match vortex lines of $\mathbf{v}$, as desired.

Our choice for $\phi$ is

$$
\begin{aligned}
\phi(\lambda, \mu)= & \phi_{e}\left(\lambda-\frac{1}{\sqrt{2}}, \mu\right) \phi_{e}\left(\lambda, \mu-\frac{1}{\sqrt{2}}\right) \\
& \times \phi_{e}\left(\lambda+\frac{1}{\sqrt{2}}, \mu\right) \phi_{e}\left(\lambda, \mu+\frac{1}{\sqrt{2}}\right),
\end{aligned}
$$

with

$$
\phi_{e}(\lambda, \mu)=\frac{(\lambda+i \mu)}{\sqrt{\lambda^{2}+\mu^{2}}} \tanh \left(\frac{\sqrt{\lambda^{2}+\mu^{2}}}{\sqrt{2} \xi}\right) .
$$

As $\phi_{e}$ has one simple zero at the origin, $\phi$ will have four simple zeros in the region $[0, \pi] \times[0, \pi]$, resulting in four nodal lines in three-dimensions. But as the circulation of 


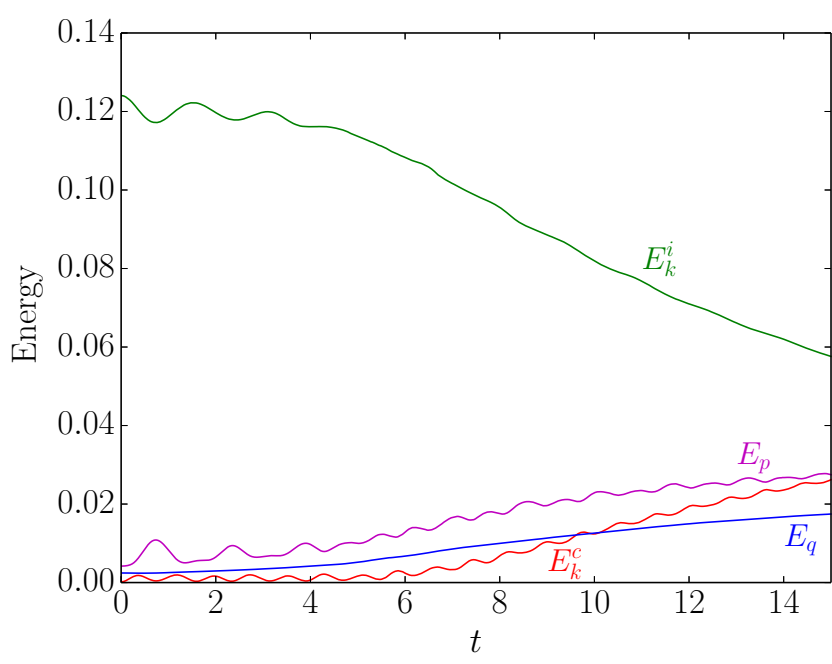

FIG. 5. Time evolution of the incompressible kinetic energy $E_{k}^{i}$, of the compressible kinetic energy $E_{k}^{c}$, of the potential energy $E_{p}$, and of the quantum energy $E_{q}$.

the velocity $\mathbf{v}$ must match the circulation generated by the vortex lines (whose circulation is in turn quantized and fixed by the parameters of the simulation), we must change the multiplicity of the nodal lines to match both values. Using that the total circulation of $\mathbf{v}$ is $\Gamma=8$, that the quantum of circulation is $4 \pi \alpha$ (with $\alpha=\hbar / 2 m$ ), and that we want multiples of four nodal lines, we write

$$
\psi(x, y, z)=\varphi(x, y, z)^{\{1 /(2 \pi \alpha)\}},
$$

where the brackets \{\} denote the integer part.

This wavefunction is then evolved in time for over 30 turnover times under the dynamics of the Advective Real Ginzburg-Landau Equation (ARGLE),

$$
\begin{array}{r}
\frac{\partial \psi}{\partial t}=\frac{c \xi}{\sqrt{2}} \nabla^{2} \psi+ \\
\frac{c}{\sqrt{2} \xi}\left(|\psi|^{2} \psi-\frac{|\psi|^{4}}{2}\right) \\
-i \mathbf{v} \cdot \nabla \psi-\frac{\sqrt{2} \mathbf{v}^{2}}{4 c \xi} \psi .
\end{array}
$$

This allows us to reduce the acoustic energy present in $\psi$, as solutions of the ARGLE converge to states which are solutions of the GPE with minimal energy in acoustic modes. Also, evolving the system under the ARGLE improves the matching of the circulations. The resulting wavefunction is finally used as the initial condition for the GPE.

\section{Appendix B: Analysis for helical perturbations}

As a reference, and for comparison with the spectra presented in Sec. IV for the Taylor-Green initial conditions, we present here the space-time resolved spectrum for a system of only four straight vortices at rest, perturbed with small helical perturbations so as to excite linear Kelvin waves. The spatial resolution and parameters of the simulation were the same as in the simulation in the main text. Initial conditions were prepared as in 24], and the vortices were perturbed with a superposition of small helical displacements between wavenumbers $k=3$ and $k=30$ (see also 24] ). The resulting spacetime resolved mass density spectrum is shown in Fig. 6 . The perturbation excites strong sound waves, but modes compatible with the dispersion relation of Kelvin waves can be identified in the range of wavenumbers excited by the small initial perturbation. Moreover, the dispersion relation of these modes is compatible with the one observed in the main text for the fully developed turbulent flow.

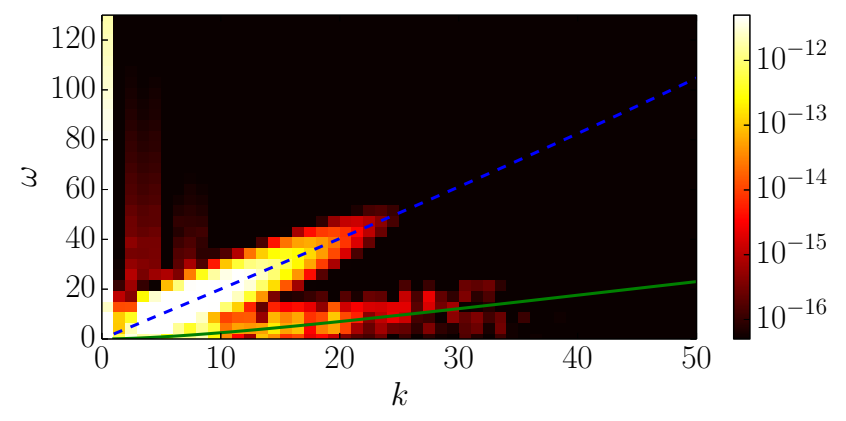

FIG. 6. Space-time resolved mass density spectrum $\rho(k, \omega)$ for a simulation with four straight vortices perturbed with small helical displacements between $k=3$ and $k=30$. The dashed (blue) line indicates the sound wave dispersion relation $\omega_{B}(k)$, and the solid (green) line indicates the Kelvin wave dispersion relation $\omega_{K}(k)$.

\section{ACKNOWLEDGMENTS}

The authors acknowledge support from Grant No. ECOS-Sud A13E01. PCdL and PDM acknowledge support from Grant Nos. PIP 11220090100825, UBACYT 20020110200359, PICT 2011-1529 and PICT 2011-1626. PDM acknowledges fruitful discussions with C. Rorai, E. Calzetta, and V. Bekeris.
[1] W. F. Vinen and J. J. Niemela, J. Low Temp. Phys. 128, 167 (2002)

[2] L. Skrbek and K. R. Sreenivasan, Phys. Fluids 24, 011301 (2012)
[3] R. J. Donnelly, Quantized Vortices in Helium II (Cambridge University Press, 1991).
[4] J. Maurer and
P. Tabeling, Europhys. Lett. 43, 29 (1998) 
[5] J. Salort, B. Chabaud, E. Lévêque, and P.-E. Roche, Europhys. Lett. 97, 34006 (2012)

[6] M. Paoletti, M. Fisher, K. Sreenivasan, and D. Lathrop, Phys. Rev. Lett. 101, 154501 (2008).

[7] A. C. White, C. F. Barenghi, N. P. Proukakis, A. J. Youd, and D. H. Wacks, Phys. Rev. Lett. 104, 075301 (2010)

[8] W. Thomson, Philos. Mag. 10, 155 (1880)

[9] E. Kozik and B. Svistunov, Phys. Rev. Lett. 92, 035301 (2004).

[10] V. S. L'vov and S. Nazarenko, J. Exp. Theor. Phys. Lett. 91, 428 (2010).

[11] L. Boué, R. Dasgupta, J. Laurie, V. L'vov, S. Nazarenko, and I. Procaccia, Phys. Rev. B 84, 064516 (2011)

[12] L. Boué, V. S. L'vov, Y. Nagar, S. V. Nazarenko, A. Pomyalov, and I. Procaccia, Physical Review B 91, 144501 (2015)

[13] C. Nore, M. Abid, and M. Brachet, Phys. Rev. Lett. 78, 3896 (1997)

[14] W. Vinen, M. Tsubota, and A. Mitani, Phys. Rev. Lett. 91, 135301 (2003).

[15] D. P. Meichle, C. Rorai, M. E. Fisher, and D. P. Lathrop, Phys. Rev. B 86, 014509 (2012).

[16] T. Maxworthy, J. Atmos. Sci. 30, 1717 (1973)

[17] D. Kleckner and W. T. M. Irvine, Nature Phys. 9, 253 (2013),

[18] S. Chen and R. H. Kraichnan, Phys. Fluids A 1, 2019 (1989).

[19] P. Clark di Leoni, P. J. Cobelli, P. D. Mininni, P. Dmitruk, and W. H. Matthaeus, Phys. Fluids 26, 035106 (2014)

[20] E. Fonda, D. P. Meichle, N. T. Ouellette, S. Hormoz, and D. P. Lathrop, Proc. Natl. Acad. Sci. U.S.A. 111, 4707 (2014)

[21] S. Nazarenko, Wave Turbulence, 2011th ed. (Springer, 2011).

[22] S. K. Nemirovskii, J. Low Temp. Phys. 171, 504 (2012)

[23] A. Baggaley and C. Barenghi, Phys. Rev. B 83, 134509 (2011)

[24] G. Krstulovic, Phys. Rev. E 86, 055301 (2012)

[25] A. W. Baggaley and J. Laurie, Phys. Rev. B 89, 014504 (2014)

[26] L. Kondaurova, V. L'vov, A. Pomyalov, and I. Procaccia, Phys. Rev. B 90, 094501 (2014)

[27] N. G. Berloff, M. Brachet, and N. P. Proukakis, Proc. Natl. Acad. Sci. U.S.A. 111, 4675 (2014).
[28] V. Bretin, P. Rosenbusch, F. Chevy, G. Shlyapnikov, and J. Dalibard, Phys. Rev. Lett. 90, 100403 (2003)

[29] A. L. Fetter, Phys. Rev. A 69, 043617 (2004)

[30] T. P. Simula, T. Mizushima, and K. Machida, Phys. Rev. Lett. 101, 020402 (2008)

[31] T. P. Simula and K. Machida, Phys. Rev. A 82, 063627 (2010).

[32] K. Schwarz, Phys. Rev. B 31, 5782 (1985)

[33] C. Nore, M. Abid, and M. E. Brachet, Phys. Fluids 9, 2644 (1997).

[34] M. Kobayashi and M. Tsubota, Phys. Rev. Lett. 94, 065302 (2005).

[35] J. Yepez, G. Vahala, L. Vahala, and M. Soe, Phys. Rev. Lett. 103, 084501 (2009)

[36] B. Rousset, P. Bonnay, P. Diribarne, A. Girard, J. M. Poncet, E. Herbert, J. Salort, C. Baudet, B. Castaing, L. Chevillard, F. Daviaud, B. Dubrulle, Y. Gagne, M. Gibert, B. Hébral, T. Lehner, P.E. Roche, B. Saint-Michel, and M. B. Mardion, Review of Scientific Instruments 85, 103908 (2014)

[37] S. Nazarenko and M. Onorato, Physica D 219, 1 (2006)

[38] D. Proment, S. Nazarenko, and M. Onorato, Phys. Rev. A 80, 051603 (2009).

[39] N. P. Proukakis and B. Jackson, Jour. Phys. B 41, 203002 (2008)

[40] S. K. Nemirovskii, Physics Reports Quantum Turbulence: Theoretica

[41] G. Krstulovic and M. Brachet, Phys. Rev. E 83, 066311 (2011).

[42] P. Mininni, D. Rosenberg, R. Reddy, and A. Pouquet, Parallel Computing 37, 316 (2011).

[43] J. Clyne, P. Mininni, A. Norton, and M. Rast, New Journal of Physics 9, 301 (2007)

[44] P. H. Roberts, Proc. Roy. Soc. Lond. A 459, 331 (2003).

[45] A. S. Bradley and B. P. Anderson, Phys. Rev. X 2, 041001 (2012)

[46] G. Baym, Phys. Rev. Lett. 91, 110402 (2003)

[47] I. Coddington, P. Engels, V. Schweikhard, and E. A. Cornell, Phys. Rev. Lett. 91, 100402 (2003)

[48] T. Mizushima, Y. Kawaguchi, K. Machida, T. Ohmi, T. Isoshima, and M. M. Salomaa, Phys. Rev. Lett. 92, 060407 (2004). 